\title{
A Simple Approach to Explain Stability of Cultural Communication and Timeliness of Media
}

\author{
Lingjin Kong $^{1 \text { a }}$, Jian Zhou ${ }^{2, b^{*}}$, Jing Zhao ${ }^{1, c}$ and Tingkui Ma ${ }^{1, d}$ \\ ${ }^{1}$ College of Journalism and Communication, Northwest Minzu University, Lanzhou, China \\ ${ }^{2}$ College of Electrical Engineering, Northwest Minzu University, Lanzhou, China \\ akonglingjin928@126.com, bzhoujianfrank@126.com, '545137473@qq.com, d540981826@qq.com \\ * The corresponding author
}

Keywords: Cultural Communication; Timeliness; Stability; Media

\begin{abstract}
This paper introduces the local area of cultural spreading is studied in the model of the path length in complex networks, and the timeliness and stability of the media with different topologies analyzed. Taking into account of the study and imitative behaviors to the other gamers in the information structure, it carries on the simulation experiment, and gets the conclusion that small-world effects have positive impact on the information communication and gambling result. Even now, this trend is still very explicit.
\end{abstract}

\section{Introduction}

The media and the information structure have showed diversified tendency, which are caused by the rapid development of the Internet and the optimization tendency of the media convergence [1-3]. In the past, the information need a lot of time to approve, hence, the transmission speed of traditional media is slow with the delay and stronger stability owing to editing, layout, printing, distribution and many other factors [4]. The development of media technology gave birth to a new medium, the emergence of new media, especially the Internet, which are making the traditional media has been an unprecedented impact and challenges [5-6]. When many of the major news at home and abroad, especially sudden incidents, the previous traditional media is no longer the first of information released to the public, replaced by new media [7]. The new media has brought the revolutionary changes of transmission history, it is not only provided with a new platform for information release, reception and transmission to the public and eliminated the time and space constraints of information, realized real-time transmission of information, but also impacted the traditional types of information production and transmission, which are changed the audience to receive information from the one-way communication situation [8-10]. Therefore, the audience can use the new media for free exchange and discussion with a strong interactivity. Nowadays, the advances in information technology has made every has the ability of information production and dissemination, which are making the information is no longer a unique existence for professional media organization, this also means that the traditional media has lost the power of information monopoly, which are caused people are in a free, tolerant media environment and enjoy a new experience that is due to information explosion, thus we must be aware of that marked the era of everyone is a disseminator has arrived [11]. Timeliness is a primary factor to achieve the value of news. The diversity of the disseminator indeed can improve the timeliness of cultural information dissemination, which could reduce the stability of information dissemination in some sense. The construction of multiple avenues can provide various options to the audience, which causes that how accurately and effectively retrieve information from the mass of information and how to produce a stable and sustainable development influence required becomes a worthy focus of concern [12-13]. In this study, we will build a model of cultural information transmission and make data analysis, it can help us conduct a thorough research on this phenomenon, and then promote the effective dissemination of cultural information. Recently, the information spreading model based on complex 
${ }^{1}$ networks theory and its begins to attract increasing attention of the interdisciplinar, for instance, SIR, SI, SIS and so on [13]. According to the previous research and setting, in order to figure out the equilibrium relationship between the stability and timeliness of the cultural information communication, this study explores the agent simulation model based on complex dynamical network, which have various state nodes [14]. The key idea of our approach is make a contribute to a deeper understanding of the behavior, which are included of the cultural information communication and efficiency of the information spreading [15], in addition, this study can offer a model and reference data for predicting the direction and scale of public opinion in the process of public opinion formation and propagation. Subsequently, the new media expedites new culture, the new culture still produce opposite effect on media and its contents. Keeping the balance, which are the media dynamical spreading and the cultural communication in the process of cultural communication [15-16].

\section{Regular Cultural Transmission Networks}

In order to understand the relationships between the topology and dynamics of a complex network, it is necessarily study and comprehend the structural characteristics of real-world cultural transmission networks. thereby establishing approximate mathematical network models. Many case studies on various real-world cultural transmission networks have been carried out and reported from different perspectives. The name regular network here does not necessarily refer to the mathematically defined concept of regular graph. One of the most typical regular networks is a fully-connected network. In such a network, between every pair of nodes there exists an edge connecting them together.

As mentioned above, a regular ring-shaped network has a large clustering coefficient and an ER random graph network has a short average path length. There are some kinds of transmission networks that have both features of large clustering along with short average path lengths, which are the so-called small transmission world networks discovered by Watts and Strogatz in 1998.

For the WS transmission network model, the clustering coefficient is redefined to be the ratio of the mean number of edges among the neighbors of a node and the number of all possible edges among the neighbors of the node:

$$
C_{\text {ws }}=\frac{N_{\text {nodes }}}{M_{\text {lengh }}}
$$

Note that $N_{\text {nodes }}$ is the average number of neighboring edges and $M_{\text {length }}$ is the total possible number of all transmission neighboring nodes.

For large enough size N, the clustering coefficient of the WS small-world transmission network model is given by as follows:

$$
C(p)=\frac{3(K-1)}{2(2 K-1)}(1-p)^{3}
$$

And the clustering coefficient of the NW small-world culture transmission network model is also given by as follows:

$$
C(p)=\frac{3(K-2)}{4(K-1)+4 K p(p+2)}
$$

It is noticeable that all the above-studied ER random-graph transmissions network model and WS/NW small world network models do not grow in size, but most real-world networks grow and indeed grow very fast. For instance, journals have new papers being published every month and WWW of the culture transmission has new websites being added every day. Subsequently, several 
research show that there are a lot of approach attempt to figure out the database of SCI. Asymptotically, we model a virtual structure of the complex networks and cultural communication system. The process starts of the initial stage of the model, in order to avoid some noise, we deliberately to set the function on the field of real numbers. Moreover, by adjusting two parameters in the virtual model, it is easily to find that we can accurately reproduce the data from the software of SPSS, in the growth of such a network, a forthcoming node has the tendency to connect itself to some "big" nodes (with large degrees), which are more important therefore more attractive in some sense. As shown in Fig. 1 and Fig. 2:

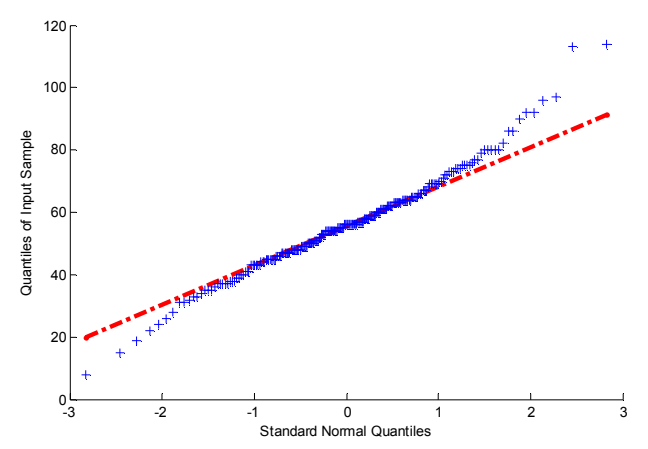

Figure 1. Plot of simulation and real collected data

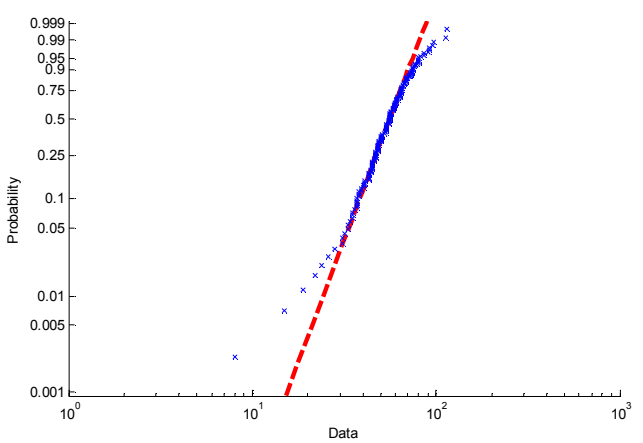

Figure 2. Plot of Weibull distribution

Table 1 Effectiveness and Stability of the Culture Transmission Network

\begin{tabular}{|c|c|c|c|}
\hline Type & Stability (\%) & Effectiveness (\%) & Error Propagation (\%) \\
\hline Real Networks & 41.97 & 31.36 & 10.61 \\
\hline Model Networks & 48.13 & 55.32 & -7.19 \\
\hline
\end{tabular}

The real data collected from Oregon route server, the looking Glass site, and the Internet Routing Registry (IRR), reveal that the Internet continuously has additions (births) and deletions (deaths) of AS and their peering relations. It can be seen that the birth rates of nodes of models and edges are both larger than their death rate, at least during this period of time.

Table 2 Average measures of the Internet at the IR, AS and EAS levels

\begin{tabular}{|c|c|c|c|c|c|c|}
\hline Level & $N$ & $E$ & $<\mathrm{k}>$ & $C$ & $L$ & $B$ \\
\hline IR & 228263 & 320149 & 2.8 & 0.03 & 9.5 & 5.3 \\
\hline AS & 11174 & 23409 & 4.2 & 0.30 & 3.6 & 2.3 \\
\hline EAS & 11461 & 32730 & 5.7 & 0.39 & 3.6 & 2.3 \\
\hline
\end{tabular}

By examining a BGP table that matches IP addresses to their corresponding AS numbers, one can label routers or interfaces with the AS number to which they belong. In this way, geographic information of the AS-level Internet infrastructure can be obtained. It is found that the number of 
distinct locations spanned by an AS is strongly correlated with the degree of the AS. For a small AS, these locations show a wide variability in the dispersal, however, for a large AS whose size exceeds a certain threshold, these locations are rapidly dispersed geographically. Based on all the above observations and discussions, referred to as the GeoBA model, is summarized as follows:

Consider a culture transmission network on a plane of linear size L, and divide it in to squares of size $l \times l$ with $1<<$ L.

Assign to each square a population density $\rho(x, y)$ with a fractal dimension.

The new node will bring in $\mathrm{m}$ new edges, the probability of a new edge connecting to an existing node $\mathrm{j}$ of degree $k_{j}$ at distance ${ }^{d_{i j}}$ from node $\mathrm{i}$.

\section{Discussion}

In this study, we presented a special analysis approach for culture transmission in complex networks. Aiming at the limitation of the above algorithm, new social networking information and cultural transmission are divided into two sections, which are a society to another society and a group to another group. In the real world, the process of cultural distribution depends on the practical value of the culture, the degree of difficulty, the reputation of civilization, the adaptability of the times and the resistance and other factors. Usually a region in the cultural characteristics and another region there is a high similarity, you can infer the spread of its foreign culture is greater than the creative capacity of the region's culture. The key idea of our approach is that make a new index to balance the stability and effectiveness of the cultural transmission network. From the results proposed and analyze, we can make a conclusion that the stability of the cultural transmission in complex network based on the quality and timeliness of the media. We can use several different indexes to balance the stability of the nodes from different nonlinear-networks.

\section{Acknowledgements}

This work is supported by Scientific Research Innovation Subject of Northwest Minzu University (Grant Yxm2016084), by Fundamental Research Funds for the Central Universities (Grant 31920160003), and by Research Funds for New Silk Road Economic Belt of Northwest Minzu University (Grant xsczl201602).

\section{References}

[1] J. Lorenz, Consensus strikes back in the Hegselmann-Krause model of continuous opinion dynamics under bounded confidence, Journal of Artificial Societies and Social Simulation, 2006, 9 (1): 8-22.

[2] K. Sznajd-Weron, Sznajd model and its applications, Acta physica polonica B, 2005, 36(8): 2537-2547.

[3] J.X. Xi, M. He, H. Liu, J.F. Zheng, Admissible output consensualization control for singular multi-agent systems with time delays, Journal of the Franklin Institute-Engineering and Applied Mathematics, 353 (2016), pp. 4074-4090.

[4] N. Cai, C. Diao, M.J. Khan, A novel clustering method based on quasi-consensus motions of dynamical multi-agent systems, Complexity, 2017, 4978613.

[5] M. Cipriani, A. Guarino, Herd behavior in financial markets: An experiment with financial market professionals, Journal of the European Economic Association, 2009, 7(1): 206-233.

[6] C. Hott, Herding behavior in asset markets, Journal of Financial Stability, 2009, 5(1): 35-56.

[7] A. Clauset, Finding Local Community Structure in Networks, Phys Rev E, 2005, 72(2): 72.026132.

[8] B. Celen, S. Kariv, Distinguishing informational cascades from herd behavior in the laboratory, American Economic Review, 2004, 94(3): 484-498. 
[9] M. E. Newman, The Structure of Scientific Collaboration Networks, The National Academy of Sciences of the United States of America, 2001, 98(2): 404-409.

[10] G. Palla, I. Derenyi, I. Farkas, T. Vicsek, Uncovering the Overlapping Community Structure of Complex Networks in Nature and Society, Nature, 2005, 435(7043): 814-818.

[11]T.C. Russo, J. Koreten, Prestige, Centrality and Learning: A Social Network Analysis of an Online Class, Communication Education, 2005, 54(3): 254-261.

[12]N. Cai, M.J. Khan, Almost decouplability of any directed weighted network topology, Physica A, 436 (2015), pp. 637-645.

[13]H.W. Ma, A.P. Zeng, the Connectivity Structure, Giant Strong Component and Centrality of Metabolic Networks, Bioinformatics, 2003, 19(11): 1423-1430.

[14]I.X.Y. Leung, H. Pan, P. Lio, J. Crowcroft, Towards real-time Community Detection in Large Networks, Physical Review E, 2009, 79(6): 79.066107.

[15]N. Cai, J. Cao, H. Ma, C. Wang, Swarm stability analysis of nonlinear dynamical multi-agent systems via relative Lyapunov function, Arab. J. Sci. Eng., 39 (2014), pp. 2427-2434.

[16]D.B. Chen, M.S. Shang, Z.H. Lv, Y. Fu, Detecting Overlapping Communities of Weighted Networks Via a Local Algorithm, Physica A: Statistical Mechanics and its Applications, 2010, 389(19): 4177-4187. 